\section{(2) OPEN ACCESS}

\title{
Initial combination therapy of ambrisentan and tadalafil in connective tissue disease-associated pulmonary arterial hypertension (CTD-PAH) in the modified intention-to-treat population of the AMBITION study: post hoc analysis
}

\author{
Masataka Kuwana (D , ' Christiana Blair, ${ }^{2}$ Tomohiko Takahashi, ${ }^{3}$ Jonathan Langley, \\ John G Coghlan ${ }^{5}$
}

Handling editor Josef $S$

Smolen

- Additional material is published online only. To view, please visit the journal online (http://dx.doi.org/10.1136/ annrheumdis-2019-216274)

${ }^{1}$ Allergy and Rheumatology, Nippon Medical School Graduate School of Medicine, Tokyo, Japan

${ }^{2}$ Research and Development, Gilead Sciences, Inc, Foster City, California, USA

${ }^{3}$ Medical Affairs,

GlaxoSmithKline Plc, Tokyo, Japan

${ }^{4}$ Medical Affairs,

GlaxoSmithKline Plc, Brentford, UK

${ }^{5}$ Cardiology Services, Royal Free Hospital, London, UK

Correspondence to Professor Masataka Kuwana, Allergy and Rheumatology, Nippon Medical School

Graduate School of Medicine,

Tokyo, Japan;

kuwanam@nms.ac.jp

Received 5 September 2019 Revised 25 February 2020 Accepted 25 February 2020 Published Online First 11 March 2020

Check for updates

(C) Author(s) (or their employer(s)) 2020. Re-use permitted under CC BY-NC. No commercial re-use. See rights and permissions. Published by BMJ.

To cite: Kuwana M,

Blair C, Takahashi T,

et al. Ann Rheum Dis

2020:79:626-634.

\section{ABSTRACT}

Objectives To evaluate initial combination therapy with ambrisentan plus tadalafil (COMB) compared with monotherapy of either agent (MONO), and the utility of baseline characteristics and risk stratification in predicting outcomes, in patients with connective tissue disease-associated pulmonary arterial hypertension (CTD-PAH) and the systemic sclerosis (SSC)-pulmonary arterial hypertension (PAH) subpopulation.

Methods This post hoc analysis of the Ambrisentan and Tadalafil in Patients with Pulmonary Arterial Hypertension (AMBITION) study included patients with CTD-PAH from the modified intention-to-treat population. Time to clinical failure (TtCF) was assessed by baseline characteristics, treatment assignment and risk group (low, intermediate and high) at baseline and week 16. TtCF was compared between groups using Kaplan-Meier curves and Cox proportional hazards regression modelling.

Results The analysis included 216 patients (COMB, $n=117 ;$ MONO, $n=99$ ). The risk of clinical failure was lower with COMB versus MONO (risk reduction: CTDPAH 51.7\%, SSc-PAH 53.7\%), particularly in patients with haemodynamic parameters characteristic of typical PAH without features of left heart disease and/or restrictive lung disease at baseline. The risk of clinical failure was lower with COMB versus MONO in the baseline low-risk group (HR not calculated due to no events in $\mathrm{COMB}$ ), baseline intermediate-risk group (HR $0.519,95 \% \mathrm{Cl} 0.297$ to 0.905$)$ and in the week 16 lowrisk group (HR $0.069,95 \% \mathrm{Cl} 0.009$ to 0.548 ).

Conclusions The benefit of COMB over MONO was demonstrated in patients with CTD-PAH, particularly in those with typical PAH haemodynamic characteristics at baseline. COMB is appropriate for patients categorised as low risk and intermediate risk at baseline and low risk at follow-up.

Trial registration number NCT01178073.

\section{INTRODUCTION}

Pulmonary arterial hypertension (PAH) is a progressive, life-threatening disease. ${ }^{12}$ Connective tissue disease-associated pulmonary arterial hypertension (CTD-PAH) is the second-most common aetiology; it has a range of underlying features, the most severe disease characteristics and the highest mortality of all PAH subgroups. ${ }^{3-5}$ Almost $75 \%$ of CTD-PAH

\section{Key messages}

What is already known about this subject?

- In the AMBITION study, patients with connective tissue disease-associated pulmonary arterial hypertension (CTD-PAH) benefited from initial combination therapy with ambrisentan plus tadalafil compared with either agent alone, when patients with risk factors for left heart disease were excluded.

What does this study add?

- In this post hoc analysis of the modified intention-to-treat population of the AMBITION study, initial combination therapy with ambrisentan and tadalafil provided benefit compared with initial monotherapy with either agent alone in patients with CTD-PAH or systemic sclerosis (SSc)-pulmonary arterial hypertension (PAH). The effect was most pronounced in patients with haemodynamic parameters characteristic of typical PAH without features of left heart disease and/or restrictive lung disease at baseline.

- This analysis also assessed the clinical utility of a three-parameter non-invasive risk stratification score. Overall, higher risk stratification at baseline was correlated with subsequent clinical failure events. A potential trend for lower risk of clinical failure with combination therapy compared with monotherapy in all risk groups was observed in both CTD-PAH and SSC-PAH populations. The clinical utility of a simplified risk category at follow-up (week 16) was limited, but when used, the risk of clinical failure was lower in patients undergoing combination therapy versus monotherapy in the low-risk group.

cases are related to systemic sclerosis (SSc), which has a poor prognosis compared with other CTDs, with a 3 year survival rate of just $56 \%{ }^{46-8}$

The current treatment strategy in $\mathrm{PAH}$ is based on assessment of disease severity at diagnosis, and subsequent treatment escalation based on the patient's condition. ${ }^{910}$ The 2015 European Society of Cardiology (ESC) and European Respiratory Society (ERS) 
Key messages

\section{How might this impact on clinical practice or future} developments?

- Combination therapy is appropriate for patients with typical PAH categorised as low and intermediate risk at baseline, while high-risk patients could possibly be considered for more advanced therapy. On reassessment of risk at followup, those remaining in the intermediate-risk category or deteriorating to the high-risk category may potentially benefit from escalation of therapy.

- A simplified risk stratification score at baseline may help inform disease management in patients with CTD-PAH, but further studies with larger patient populations are required.

guidelines and 2018 World Symposium of Pulmonary Hypertension consensus recommend the use of risk stratification to guide treatment decisions, classifying patients as low, intermediate or high-risk. ${ }^{10}$ However, the clinical utility of risk stratification in patients with CTD-PAH or SSc-PAH has not yet been established. ${ }^{11}$

The Ambrisentan and Tadalafil in Patients with Pulmonary Arterial Hypertension (AMBITION) study demonstrated a reduced risk of clinical failure in treatment-naive patients with $\mathrm{PAH}$ receiving initial ambrisentan plus tadalafil compared with initial monotherapy of either agent. ${ }^{12}$ A subanalysis of AMBITION and a separate clinical trial showed that combination therapy with ambrisentan and tadalafil is effective in the treatment of CTD$\mathrm{PAH}$ and SSc-PAH. ${ }^{813}$ Additionally, although SSc-PAH is typically less responsive to therapy than other forms of $\mathrm{PAH}$, significant improvements in haemodynamic measurements (such as cardiac index, mean pulmonary artery pressure (mPAP) and pulmonary vascular resistance (PVR)) were observed in response to ambrisentan plus tadalafil compared with monotherapy, suggesting that these haemodynamic measurements have prognostic value. ${ }^{8}$

The AMBITION protocol was amended during the trial to exclude patients with less pronounced haemodynamics and/ or multiple risk factors for left heart disease. ${ }^{12}$ The primary analysis set (PAS) included patients who met these amended criteria; the modified intention-to-treat (mITT) population comprised all randomised patients who received study drug, including those who did not meet the revised eligibility criteria (ex-PAS). The mITT population included an additional 29 patients with CTD-PAH, 19 of whom had SSc-PAH. Results for the PAS population ${ }^{12}$ and a post hoc analysis of the CTD-PAH and SSc-PAH subgroups in the PAS $^{13}$ have been published, as has a post hoc analysis of the mITT population, which found that initial combination therapy may be associated with improved long-term survival compared with initial monotherapy. ${ }^{14}$

This post hoc subgroup analysis of the AMBITION mITTCTD-PAH population assessed the relationship between baseline characteristics (including haemodynamic parameters) and outcome, and evaluated the utility of an abbreviated, threeparameter non-invasive risk stratification score in predicting outcomes in patients with CTD-PAH.

\section{METHODS}

\section{Study design}

This was a post hoc subgroup analysis of the mITT-CTD-PAH population and SSc-PAH subpopulation of the AMBITION study. ${ }^{12}$ Patients were randomised 2:1:1 to receive one time per day combination therapy (ambrisentan $10 \mathrm{mg}$ plus tadalafil
$40 \mathrm{mg}$ ) or monotherapy (ambrisentan $10 \mathrm{mg}$ plus placebo or tadalafil $40 \mathrm{mg}$ plus placebo). Details of the study design have been described previously. ${ }^{12}$

\section{Patient and public involvement}

Patients were not involved in the design of this study nor in the post hoc analysis.

\section{Patient population}

The patient population has been described previously. ${ }^{12} 13$ During the study, a blinded interim review revealed a high prevalence of risk factors for left ventricular diastolic dysfunction; therefore, eligibility criteria were amended so that patients with $\geq 3$ of the following risk factors for left ventricular diastolic dysfunction were excluded: body mass index $\geq 30 \mathrm{~kg} / \mathrm{m}^{2}$, history of essential hypertension, diabetes mellitus and significant coronary artery disease. In addition, inclusion criteria for the diagnosis of $\mathrm{PAH}$ were modified: PVR increased from $\geq 240$ to $\geq 300$ dyne $/ \mathrm{sec} /$ $\mathrm{cm}^{5}$; for patients with a PVR 300-499 dyne $\cdot \mathrm{sec} / \mathrm{cm}^{5}$, a pulmonary arterial wedge pressure (PAWP)/left ventricular end diastolic pressure of $\leq 12 \mathrm{~mm} \mathrm{Hg}$ was required (PAS population). ${ }^{12}$ This analysis included all patients (PAS and ex-PAS) who were randomised and received $\geq 1$ dose of study drug (mITT population).

\section{Assessments}

The AMBITION trial ${ }^{12}$ primary endpoint was the time from randomisation to the first adjudicated clinical failure. Clinical failure was defined as death (any cause), hospitalisation for worsening $\mathrm{PAH}$, disease progression (defined as a decrease of $>15 \%$ in 6 min walking distance (6MWD) from baseline combined with WHO Functional Class (WHO-FC) III/IV symptoms at two consecutive study visits $\geq 2$ weeks apart), or unsatisfactory long-term clinical response (decrease in 6MWD from baseline (at two consecutive study visits $\geq 2$ weeks apart)), together with WHO-FC III symptoms after 6 months of therapy. Secondary endpoints included change from baseline to week 24 in N-terminal pro-B-type natriuretic peptide (NT-proBNP) and 6MWD.

Time to clinical failure (TtCF) for the following baseline subgroups was investigated (median values were used for continuous variables): age ( $\leq />63$ years); SSc-PAH diagnosis (yes/ no); haemodynamic parameters (mPAP $(\leq />43 \mathrm{~mm} \mathrm{Hg})$ ); PVR $\left(\leq />593.5\right.$ dyne $\left.\cdot \mathrm{sec} / \mathrm{cm}^{5}\right)$; PAWP $(\leq />9 \mathrm{~mm} \mathrm{Hg})$; cardiac index $\left(\leq />2.5 \mathrm{~L} / \mathrm{min} / \mathrm{m}^{2}\right)$; transpulmonary pressure gradient (TPG, $\leq />34 \mathrm{~mm} \mathrm{Hg}$ )); pulmonary function test parameters (forced expiratory volume in one second $\left(\mathrm{FEV}_{1}, \%\right.$ predicted normal) $\leq / \geq 79.025 \%$ ); total lung capacity (TLC, \% predicted normal) $\leq />86.11 \%) ; \geq 1$ comorbidity or no comorbidities, included in the PAS population (yes/no); immunosuppressant use (yes/no); and abbreviated risk category (low, intermediate or high). These parameters were selected as they have been identified as predictors of poor outcome in prior studies. ${ }^{1011} 15-18$

This study also assessed the clinical utility of an abbreviated version of the risk assessment method proposed in the 2015 ESC/ERS PAH guidelines. ${ }^{10} 19$ Risk score was determined at baseline and week 16 based on three parameters graded 1 (low risk), 2 (intermediate risk) or 3 (high risk), according to thresholds defined in the $2015 \mathrm{ESC} / \mathrm{ERS} \mathrm{PAH}$ guidelines ${ }^{10}$ : $6 \mathrm{MWD}$ (low, >440 m; intermediate, 165-440 m; and high, $<165 \mathrm{~m}$ ), NT-proBNP (low, <300 ng/L; intermediate, 300-1400 ng/L; and high, $>1400 \mathrm{ng} / \mathrm{L}$ ) and WHO-FC (low, I or II; intermediate, III; and high, IV). This grading method was based on a previous analysis of the Comparative, Prospective Registry of Newly Initiated Therapies for Pulmonary Hypertension (COMPERA) 
database. ${ }^{20}$ A mean grade was obtained by dividing the sum of all grades by the number of available parameters for each patient/ time point and rounded to the closest integer. Transition from baseline to week 16 was categorised as improved, maintained or exacerbated. Patients in the high-risk subgroup at baseline and week 16 were included in the exacerbated group. TtCF after week 16 was analysed by week 16 abbreviated risk score category. Week 16 was selected rather than week 24 to minimise the number of events excluded. Risk stratification in the CTD-PAH population was also assessed according to the French registry non-invasive method (ie, stratification by number of ESC/ERS low-risk criteria ${ }^{10}$ fulfilled for WHO-FC, NT pro-BNP and 6MWD) at baseline and week $16 .^{19}$

Safety was assessed through incidence of adverse events (AEs). Efficacy and safety assessments were performed at screening and randomisation visits, at weeks 4, 8, 16, 24 and every 12 weeks thereafter, at the final assessment visit and at the end-of-study visit.

\section{Statistical analysis}

The Kaplan-Meier product limit method was used to generate curves for TtCF. HRs and associated 95\% CIs were calculated using Cox proportional hazards regression. For the subgroup analyses, the regression model included a term for subgroup by treatment interaction. All analyses are post hoc; therefore, $\mathrm{p}$ values are not presented, except where a HR cannot be calculated due to no clinical failure events. For the Cox proportional hazards regression, where there was evidence of non-proportional hazards, restricted mean survival time was calculated based on time from randomisation to first clinical failure event or week 48 (area under the curve for each group). Week 48 was selected as the time point because it provided a sufficient sample size. For $6 \mathrm{MWD}$, missing values when calculating change from baseline to week 24 were imputed using last-observation-carried-forward imputation or worst-case imputation. ${ }^{12}$

\section{RESULTS}

\section{Study population}

This analysis included 216 patients with CTD-PAH (combination therapy, $n=117$; monotherapy (pooled), $n=99$ ) from the mITT population $(n=605)$ (figure 1$)$. Most patients were female and age was higher in the SSc-PAH group than in the CTD-PAH group; other disease characteristics were similar (table 1).

\section{Time to clinical failure}

The risk of clinical failure was $51.7 \%$ lower in the combination therapy group than in the monotherapy group in the CTD-PAH population (HR 0.483 , 95\% CI $0.286 \%$ to $0.817 \%$ ) and $53.7 \%$ lower in the SSc-PAH population (HR $0.463,95 \%$ CI $0.240 \%$ to $0.895 \%$ ) (figure 2).

\section{Change in NT-proBNP and 6MWD from baseline to week 24}

In CTD-PAH and SSc-PAH populations, the decrease in geometric mean NT-proBNP levels and the increase in 6MWD from baseline to week 24 were greater in patients receiving combination therapy than monotherapy (online supplementary table S1).

\section{TtCF by baseline subgroup}

In the CTD-PAH population, a trend for lower risk of clinical failure with combination therapy versus monotherapy was observed for all baseline subgroups, except PVR $\leq 593.5$ dyne $\cdot \mathrm{sec} / \mathrm{cm}^{5}$ and ex-PAS; however, data should be interpreted cautiously due to large CIs. The risk of clinical failure was lower in patients undergoing combination therapy versus monotherapy for the following baseline haemodynamic characteristics: mPAP $>43 \mathrm{~mm} \mathrm{Hg}$ : HR $0.46,95 \%$ CI 0.24 to 0.89 ; PVR $>593.5$ dyne.sec/ $\mathrm{cm}^{5}$ : HR $0.25,95 \%$ CI 0.13 to 0.51 ; TPG > 34: HR $0.35,95 \%$ CI 0.18 to 0.68 ; PAWP $\leq 9 \mathrm{~mm} \mathrm{Hg}$ : HR $0.32,95 \%$ CI 0.15 to 0.69 ; cardiac index $\leq 2.5 \mathrm{~L} / \mathrm{min} / \mathrm{m}^{2}$ : HR $0.34,95 \%$ CI 0.18 to 0.67 ; and TLC $>86.11 \%$ : HR $0.30,95 \%$ CI 0.15 to 0.61 (figure 3 ). The risk of clinical failure was also lower with combination therapy versus monotherapy in the PAS population, patients aged $\leq 63$ years, patients who were not receiving baseline immunosuppressants, patients with $\geq 1$ comorbidity and patients with no comorbidities (figure 3 ). For analyses showing evidence of non-proportional hazards (PVR >593.5 dyne.sec/ $\mathrm{cm}^{5}$, cardiac index $\leq 2.5 \mathrm{~L} / \mathrm{min} / \mathrm{m}^{2}$ and no comorbidities), restricted mean survival times are detailed in online supplementary table S2.
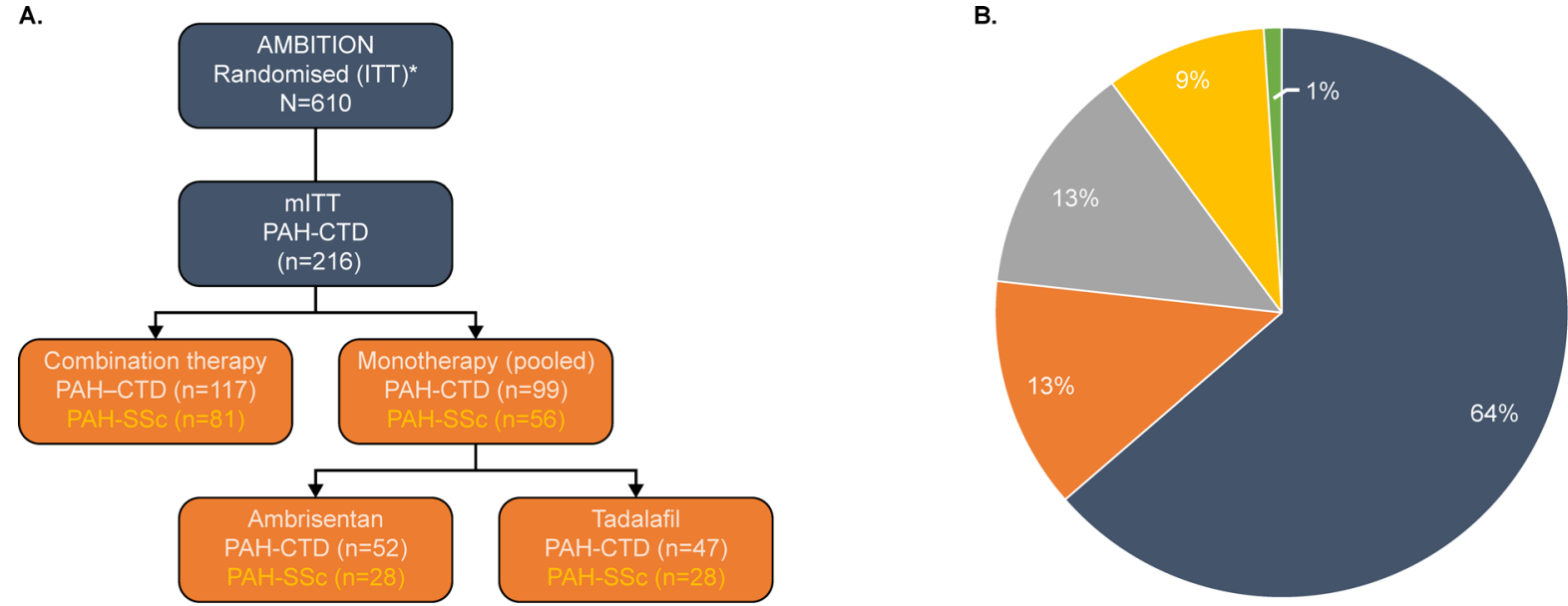

SSc Other $\square$ MCTD $\square$ SLE $\quad$ Overlap

Figure 1 Participant disposition and aetiology: (A) population distribution among treatment arms and (B) CTD-PAH population aetiologies (post hoc summary). *Five patients did not receive the study drug. CTD, connective tissue disease; ITT, intention to treat; MCTD, mixed connective tissue disease; mITT, modified intention to treat; PAH, pulmonary arterial hypertension; SSC, systemic sclerosis; SLE, systemic lupus erythematosus. 


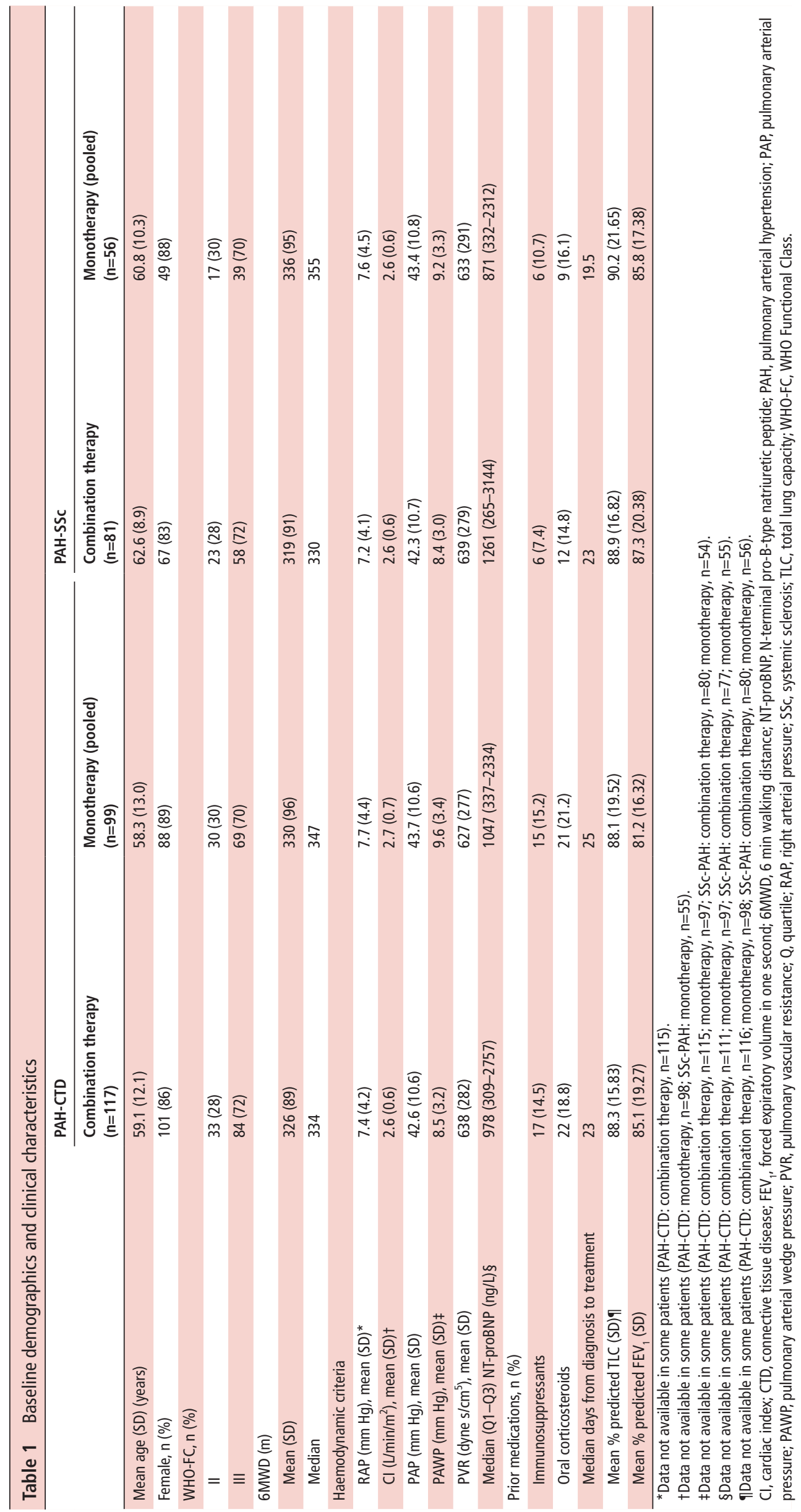


A.

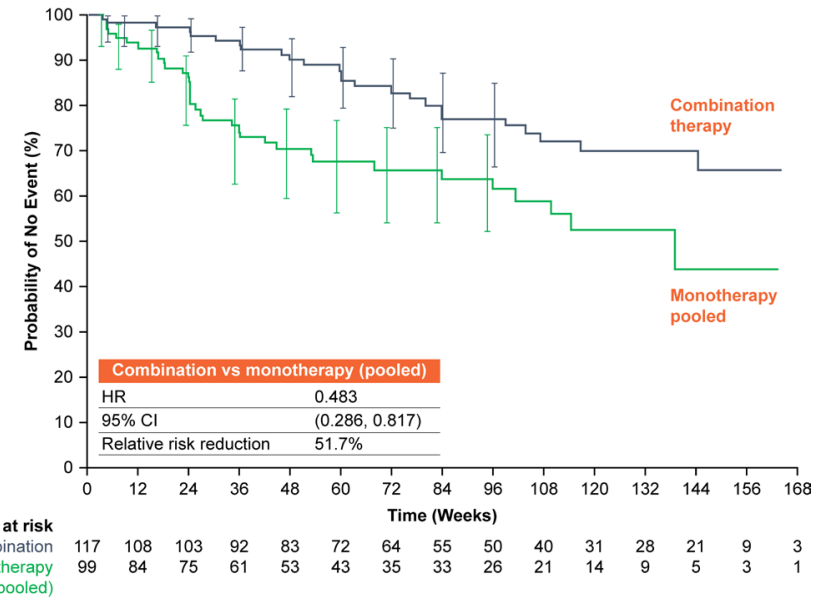

B.

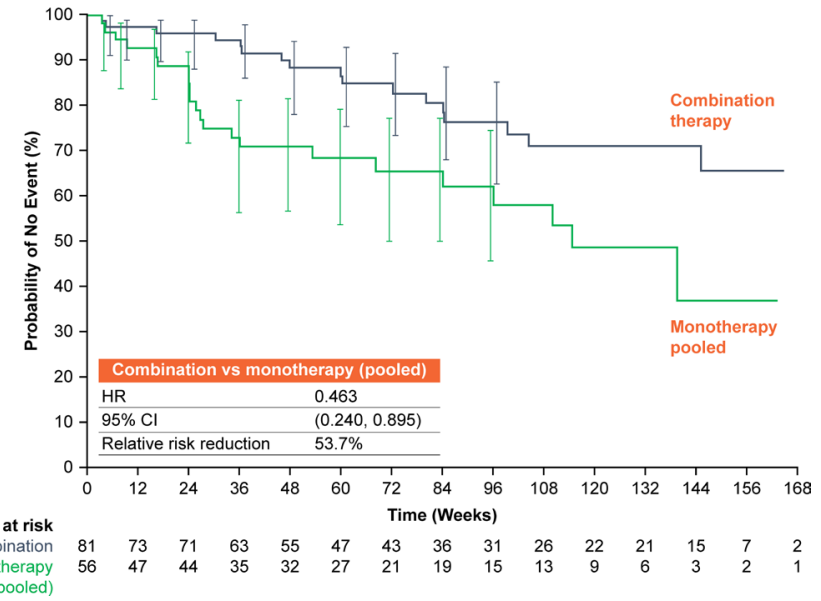

Figure 2 Kaplan-Meier plots of time to first adjudicated clinical failure in the modified intention-to-treat population: (A) connective tissue disease-associated pulmonary arterial hypertension and (B) systemic sclerosis pulmonary arterial hypertension. $95 \%$ Cls (using log-log transform method) are presented for each treatment group at weeks 4 , 8,16 and 24 , and then every 12 weeks up to week 96 .

\section{Risk stratification}

When using the abbreviated COMPERA risk stratification method, at baseline, 27/216 (12.5\%) patients were classified as low risk, $179 / 216(82.9 \%)$ as intermediate risk and 10/216 $(4.6 \%)$ as high-risk. In the CTD-PAH population, the risk of clinical failure was lowest for patients in the low-risk group and highest in the high-risk group (low vs high: HR 0.192, 95\% CI 0.047 to 0.777 ) (figure 4A). Kaplan-Meier curves demonstrated a potential trend towards lower risk of clinical failure with combination therapy versus monotherapy in all risk groups at baseline (figure 4B). The risk of clinical failure was lower with combination therapy than monotherapy in the intermediate-risk group (intermediate-risk: HR $0.519,95 \%$ CI 0.297 to 0.905 ). However, in the high-risk group, although a trend towards a lower risk of clinical failure with combination therapy versus monotherapy was observed (HR 0.197, 95\% CI 0.022 to 1.803 ), the sample sizes were small (combination therapy, $n=4$; monotherapy, $\mathrm{n}=6$ ). Additionally, in the low-risk group, no HR could be calculated due to no events in the combination therapy group; however, comparison by log-rank test supported a lower risk of clinical failure with combination therapy versus monotherapy (figure 4B).

At week 16, 19 patients had missing data; 55/197 (28\%) patients were classified as low risk, 135/197 (69\%) as intermediate risk and 7/197 (4\%) as high risk. No significant difference was observed between treatment groups in the proportion of patients who improved, maintained or worsened from baseline to week 16 (online supplementary table S3). Risk of clinical failure on or after week 16 was lowest in the low-risk group and highest in the high-risk group (low vs high: HR 0.198, 95\% CI 0.067 to 0.580 ) (figure 4C). The risk of clinical failure after week 16 was lower with combination therapy versus monotherapy for patients in the week 16 low-risk group (HR $0.069,95 \%$ CI 0.009 to 0.548 ); no significant difference between treatments was observed in the week 16 intermediate-risk group (HR $0.705,95 \%$ CI 0.372 to 1.337 ) (figure 4D). At week 16, patient numbers were insufficient in the high-risk group to allow a meaningful comparison between treatment groups.

Findings showed a similar pattern in the SSc-PAH population compared with the CTD-PAH population (online supplementary figure S1).

When assessed via the French registry non-invasive method, patients fulfilling two or three low-risk criteria at baseline or week 16 had a significantly lower risk of subsequent clinical failure than those fulfilling 0 criteria (online supplementary figure S2). The risk of clinical failure at week 16 was lower with combination therapy versus monotherapy in patients fulfilling two or three criteria (online supplementary figure S2).

\section{Safety}

Overall, there was a similar AE profile across both treatment groups in the CTD-PAH and SSc-PAH populations (table 2). The most common AEs in both treatment groups were peripheral oedema and headache. Peripheral oedema was more common with combination therapy than monotherapy (both CTD-PAH and SSc-PAH) (table 2).

\section{DISCUSSION}

In this post hoc analysis of the AMBITION study mITT population, initial combination therapy of ambrisentan and tadalafil was more effective than monotherapy in reducing the risk of clinical failure: $51.7 \%$ reduction in patients with CTD-PAH and $53.7 \%$ reduction in patients with SSc-PAH. The risk of clinical failure in the CTD-PAH population was lower with combination therapy versus monotherapy for most baseline haemodynamic characteristic subgroups.

The benefit of combination therapy over monotherapy was pronounced in patients with baseline haemodynamic parameters characteristic of typical PAH, such as high PVR, low cardiac index, high TPG and low PAWP. By contrast, in patients with an increased risk of left heart disease (low PVR and high PAWP) and/ or restrictive lung disease (low TLC), the benefit of combination therapy was less pronounced. Therefore, in the ex-PAS population (an older subgroup of patients with more cardiovascular comorbidities), ${ }^{21}$ less benefit may be gained from combination therapy. ${ }^{22}$ Further research to determine the optimal treatment approach in addition to standard PAH therapies is required for this population.

The overall treatment goal in PAH is to achieve or maintain low-risk status ${ }^{1019}$; therefore, classification of low-risk at baseline or transitioning to low-risk status at week 16 is expected to be associated with improved outcomes. ${ }^{19}$ Overall, higher risk stratification at baseline and week 16 was correlated with higher risk of clinical failure. When stratified by baseline risk group, Kaplan-Meier plots suggested a potential trend towards lower risk of clinical failure with combination therapy versus monotherapy in all risk groups (CTD-PAH and SSc-PAH populations); however, patient numbers in the high-risk groups were small. 


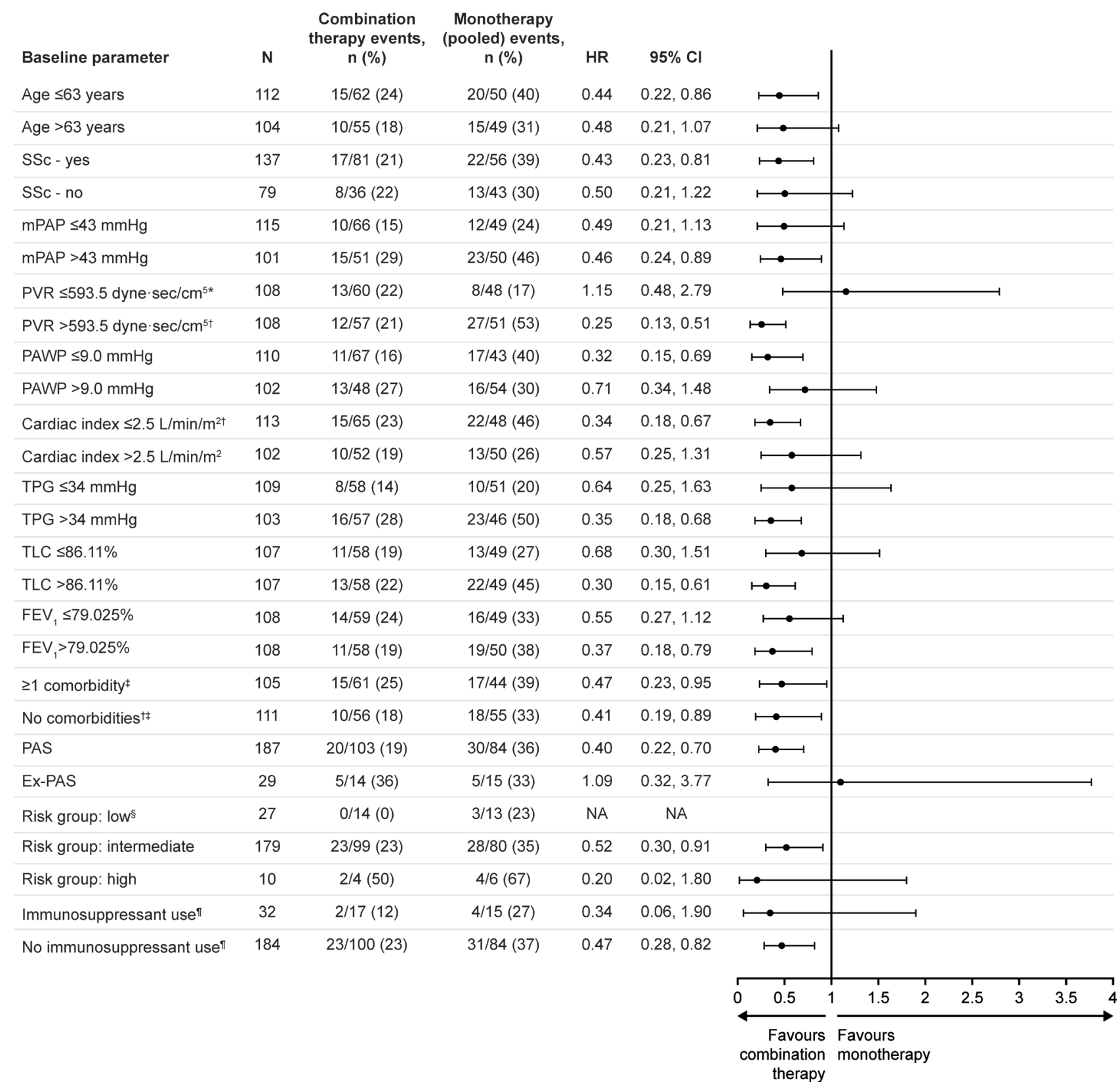

Figure 3 Forest plot of time to first adjudicated clinical failure: combination therapy versus monotherapy (pooled) per baseline subgroup. *Significant treatment by subgroup interaction; tevidence of non-proportionality (see online supplementary table S2); †hypertension, diabetes and coronary artery disease; §low-risk valid HR could not be calculated; Пindication for immunosuppressant use unknown. FEV , forced expiratory volume in one second; mPAP, mean pulmonary arterial pressure; NA, not applicable; PAS, primary analysis set; PAWP, pulmonary arterial wedge pressure; PVR, pulmonary vascular resistance; SSC, systemic sclerosis; TLC, total lung capacity; TPG, transpulmonary pressure gradient.

When stratified by risk group at week 16, Kaplan-Meier plots showed a clear benefit for low-risk patients receiving combination therapy versus monotherapy. For patients at intermediate risk, combination therapy provided a reduced risk of clinical failure versus monotherapy from week 16-100, then the lines converged and plateaued. This suggests that combination therapy may delay, but not prevent, progression in patients considered intermediate risk at follow-up; therefore, these patients and low-risk patients on monotherapy may benefit from escalation of therapy. Additionally, patients considered high risk at baseline or deteriorating to the high-risk group at follow-up may benefit from an escalation of therapy, but patient numbers were insufficient to draw meaningful conclusions. Clinical evaluation based on the maintenance or improvement of risk score using simplified risk stratification at week 16 may have limited utility in the prediction of future outcomes in this population.

Methods of risk score calculation employed in previous studies often used a higher number of invasive and/or non-invasive parameters than the method employed here. ${ }^{23-25}$ For example, a previous analysis of the AMBITION study used the Registry to Evaluate Early and Long-Term PAH Disease Management (REVEAL)risk score to stratify patients by baseline risk score. ${ }^{25}$ In the current analysis, the utility of an abbreviated COMPERA risk score using three non-invasive parameters was evaluated. This methodology ensured a direct comparison between baseline and week 16 as these non-invasive parameters were assessed at both time points. In addition, stratification using simplified, minimally invasive parameters has been shown to have a 
A.

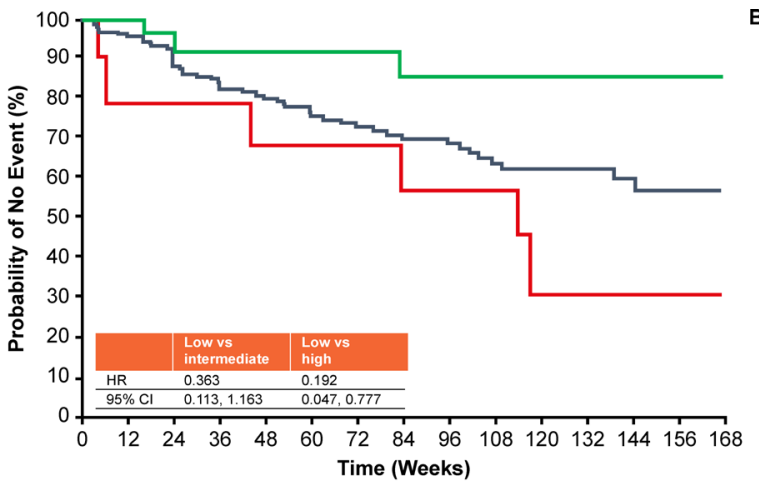

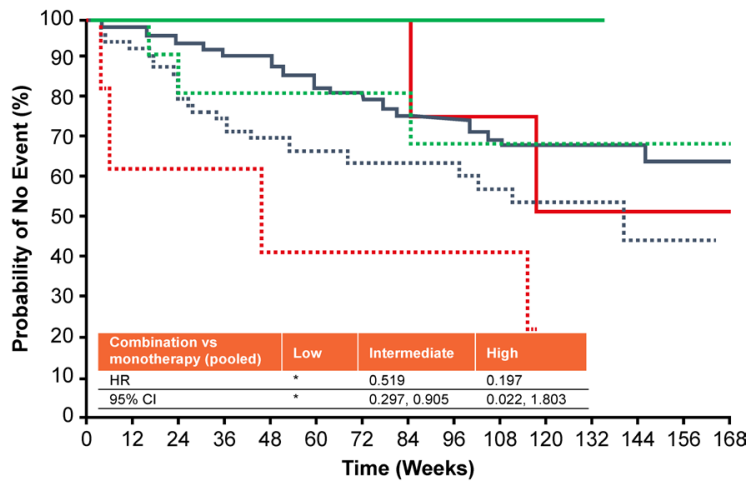

$\mathrm{N}$ at risk

- Low risk $\begin{array}{llllllllllllllll}27 & 26 & 24 & 21 & 19 & 15 & 14 & 14 & 11 & 9 & 5 & 5 & 1 & 1 & 1 & \begin{array}{l}\text { Nat risk } \\ \text { Combination }\end{array}\end{array}$

- Intermediate risk $179 \begin{array}{lllllllllllllll}159 & 147 & 125 & 111 & 94 & 79 & 68 & 60 & 47 & 38 & 30 & 23 & 10 & 2 \text { - Low risk }\end{array}$

$\begin{array}{lllllllllllllll}14 & 14 & 14 & 12 & 11 & 9 & 8 & 8 & 8 & 6 & 3 & 3 & 0 & 0 & 0\end{array}$

$\begin{array}{lllllllllllllll}99 & 90 & 85 & 76 & 68 & 59 & 52 & 43 & 39 & 31 & 26 & 23 & 19 & 8 & 2 \\ 4 & 4 & 4 & 4 & 4 & 4 & 4 & 4 & 3 & 3 & 2 & 2 & 2 & 1 & 1\end{array}$

\begin{tabular}{llllllllllllllllll}
- High risk & 4 & 4 & 4 & 4 & 4 & 4 & 4 & 4 & 3 & 3 & 2 & 2 & 2 & 1 & 1 \\
\hline M
\end{tabular}

Monotherapy (pooled)

$\begin{array}{llllllllllllllll}\text { *.. Low risk } & 13 & 12 & 10 & 9 & 8 & 6 & 6 & 6 & 3 & 3 & 2 & 2 & 1 & 1 & 1\end{array}$

... Intermediate risk $80 \quad 69 \begin{array}{llllllllllllll}80 & 49 & 43 & 35 & 27 & 25 & 21 & 16 & 12 & 7 & 4 & 2 & 0\end{array}$

c.

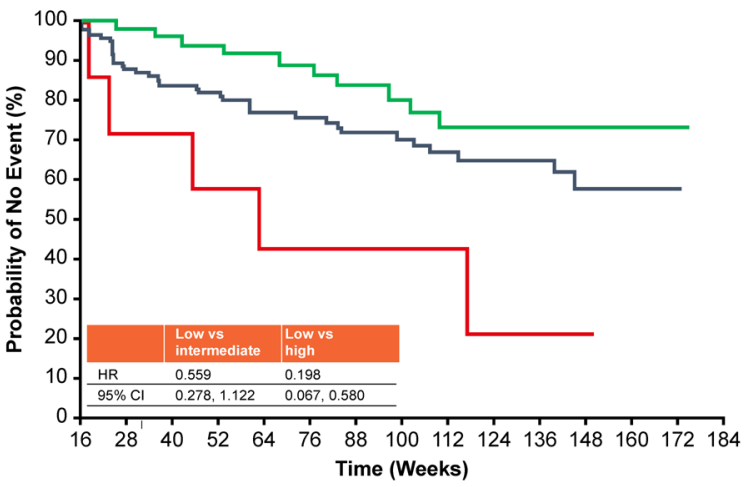

D.

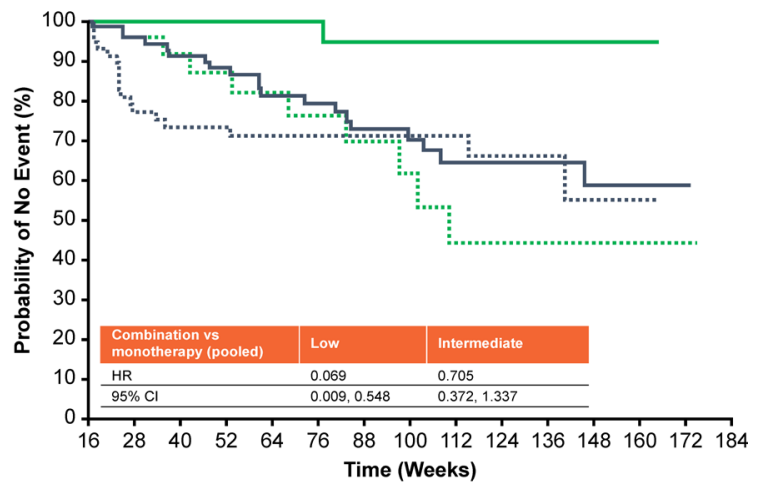

$\mathrm{N}$ at risk

- Low risk $\begin{array}{lllllllllllll}55 & 49 & 46 & 43 & 37 & 33 & 29 & 24 & 19 & 15 & 13 & 6 & 4\end{array}$

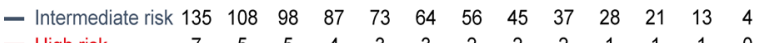

- High risk

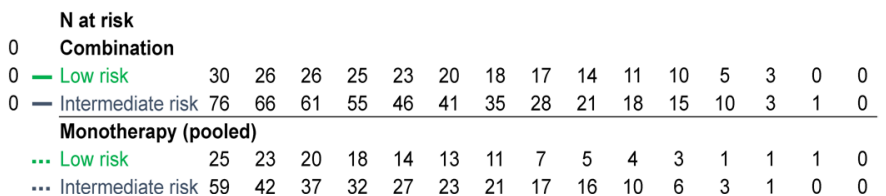

Figure 4 Kaplan-Meier curves for time to clinical failure in the CTD-PAH population by risk category according to the abbreviated COMPERA method at baseline and week 16: (A) baseline CTD-PAH: overall, (B) baseline CTD-PAH: combination versus monotherapy (pooled), (C) week 16 CTDPAH: overall and (D) week 16 CTD-PAH: combination versus monotherapy (pooled (low risk and intermediate risk only)). *NO HR calculation due to $\mathrm{NO}$ event in the combination therapy group (log-rank test: $\mathrm{p}=0.048$ ). Week 16 Kaplan-Meier plots were described based on the period from week 16 to the last assessment visit. Patient numbers were too low in the week 16 high-risk group to provide a meaningful comparison. CTD-PAH, connective tissue disease-associated pulmonary arterial hypertension.

predictive value similar to the full REVEAL risk score; ${ }^{26} 27$ this technique is likely to be simpler to apply in the clinic compared with those involving invasive parameters.

The lower number of parameters used in the current analysis may partly explain why risk categorisation did not support the benefit of initial combination therapy compared with monotherapy across all risk groups at both time points. Additionally, the non-invasive approach may not adequately discriminate low-risk and intermediate-risk patients. Given that our findings suggest the importance of baseline haemodynamic characteristics in predicting outcomes in patients with CTD-PAH, the addition of haemodynamic information may improve the sensitivity and validity of risk stratification and may help inform disease management. ${ }^{11}$ Another approach to improve risk stratification could be to use the seven-parameter stratification as per the COMPERA analysis ${ }^{24}$ for risk stratification at baseline, with the abbreviated four-parameter non-invasive method $^{23}$ at follow-up. However, in a recent post hoc analysis of the PATENT and CHEST studies, ${ }^{28-31}$ an association observed between the number of low-risk criteria achieved and improved outcomes suggested the validity of a three-parameter non-invasive risk assessment method in predicting outcomes in patients with PAH. ${ }^{19}$

When we determined risk stratification using the French registry non-invasive method, a higher number of low-risk criteria were associated with a lower clinical failure risk at baseline and follow-up; this is in line with findings using the abbreviated COMPERA method, supporting its validity as a risk assessment tool. Further studies with larger patient populations would be valuable in confirming the clinical utility of risk stratification in predicting outcomes.

AEs were consistent with the known safety profile of ambrisentan and tadalafil in the CTD-PAH population. ${ }^{12}{ }^{13}$ In line with findings in the AMBITION trial, ${ }^{12}$ peripheral oedema was more common with combination therapy than monotherapy. In patients receiving multiple therapies, a greater number of side effects compared with patients undergoing single therapy are possible, particularly therapies associated with vasodilation. ${ }^{32}$ 
Table 2 Summary of AEs in patients with CTD-PAH and SSC-PAH (mITT population)

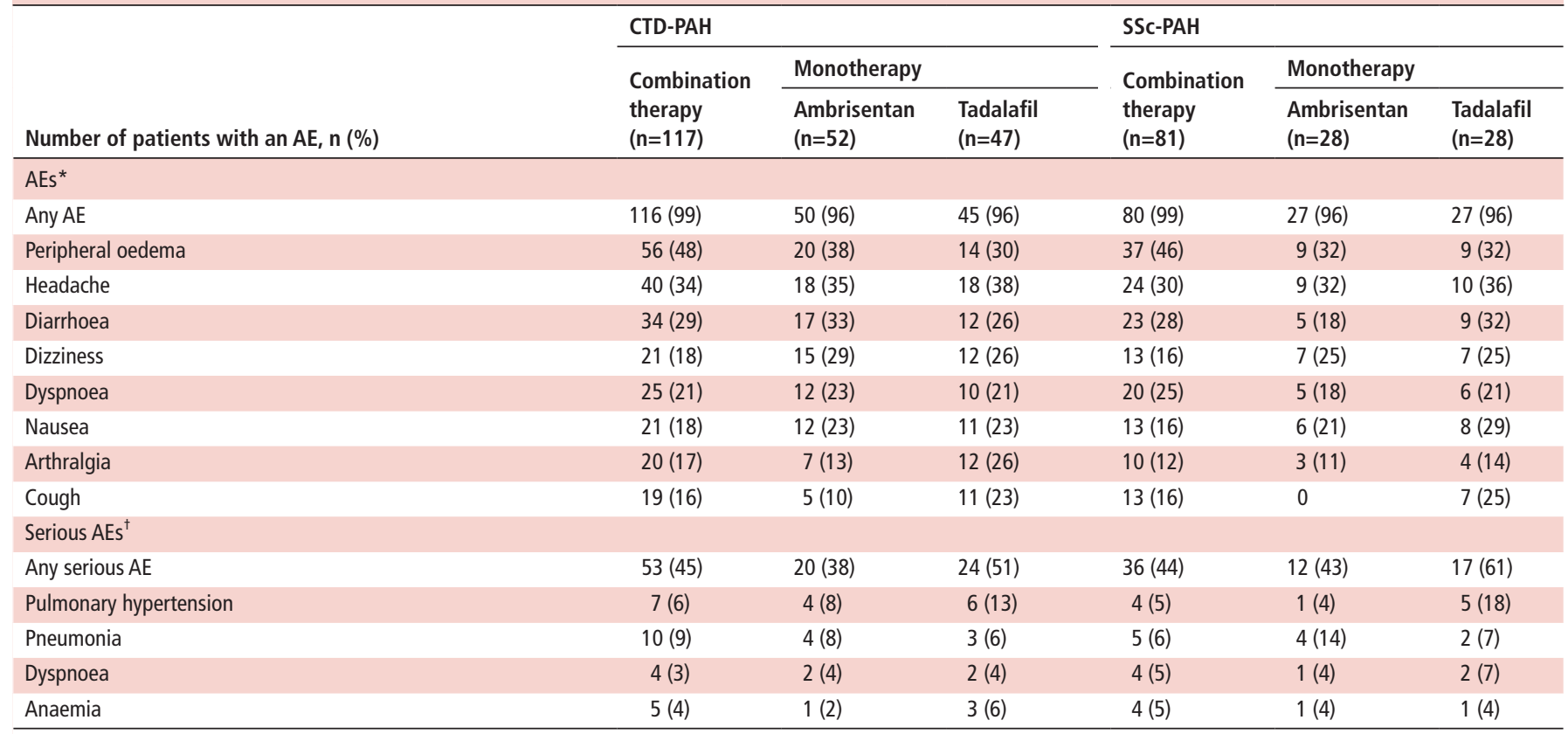

${ }^{*}$ AEs occurring in $\geq 25 \%$ of patients in any group.

tSerious AEs occurring in $>4 \%$ of patients in any group.

$\mathrm{AE}$, adverse event; CTD, connective tissue disease; mITT, modified intention to treat; $\mathrm{PAH}$, pulmonary arterial hypertension; SSC, systemic sclerosis.

In patients with CTD-PAH, the risk of clinical failure was lower with combination therapy versus monotherapy, particularly in those with baseline haemodynamic parameters characteristic of typical PAH, without features of left heart disease and/or restrictive lung disease. A simplified risk stratification score at baseline using non-invasive parameters may be useful in predicting PAH-related outcomes in patients with CTD-PAH; however, its clinical utility at week 16 is limited. At baseline, Kaplan-Meier curves demonstrated a potential trend towards lower risk of clinical failure with combination therapy versus monotherapy in all risk groups. Combination therapy appeared beneficial compared with monotherapy in the week 16 low-risk group; other groups may benefit from escalated therapy. Incorporating additional haemodynamic information may increase risk stratification validity at follow-up; further research determining the optimal predictive tools in terms of applicability and accuracy at baseline, and follow-up in a large-scale cohort is warranted.

Correction notice This article has been corrected since it published Online First. In table 1, the unit of PVR has been corrected and number of FC III in SSC/Mono amended.

Acknowledgements Medical writing support was provided by Gillian Wallace, MSc, of Fishawack Indicia Ltd, UK, and funded by GSK. The results of this study were presented in part as an oral presentation at the annual European League Against Rheumatism meeting, Madrid, 12-15 June, 2019 (Ann Rheum Dis 2019; 78:105-6) and in part at the 5th Systemic Sclerosis World Congress, Bordeaux, 15-17 February 2018 (Scleroderma Relat Disord 2018;3: suppl).

Contributors All listed authors contributed substantially to the planning and implementation of this research. All authors revised the manuscript critically for important intellectual content, approved the final version for publication and agreed to be listed as authors.

Funding This study was funded by GlaxoSmithKline (study number 112565) and Gilead Sciences, Inc

Competing interests MK has received personal fees from GSK, Bayer and Nippon Shinyaku, and research grants and personal fees from Actelion and Pfizer. $\mathrm{CB}$ is an employee and shareholder at Gilead Sciences. $\mathrm{JL}$ is a former employee and shareholder of GSK. TT is an employee at GSK. JGC has received research grants and personal fees from Actelion and GSK, and personal fees from Bayer, Endotronix, Pfizer and United Therapeutics.
Patient and public involvement Patients and/or the public were not involved in the design, or conduct, or reporting, or dissemination plans of this research.

Patient consent for publication Not required.

Provenance and peer review Not commissioned; externally peer reviewed.

Data availability statement Anonymised individual participant data and study documents can be requested for further research from www. clinicalstudydat arequest.com.

Open access This is an open access article distributed in accordance with the Creative Commons Attribution Non Commercial (CC BY-NC 4.0) license, which permits others to distribute, remix, adapt, build upon this work non-commercially, and license their derivative works on different terms, provided the original work is properly cited, appropriate credit is given, any changes made indicated, and the use is non-commercial. See: http://creativecommons.org/licenses/by-nc/4.0/.

\section{ORCID iD}

Masataka Kuwana http://orcid.org/0000-0001-8352-6136

\section{REFERENCES}

1 Frost A, Badesch D, Gibbs JSR, et al. Diagnosis of pulmonary hypertension. Eur Respir J 2018:1801904.

2 Montani D, Günther S, Dorfmüller P, et al. Pulmonary arterial hypertension. Orphanet J Rare Dis 2013;8:97

3 Prins KW, Thenappan T. World Health organization group I pulmonary hypertension: epidemiology and pathophysiology. Cardiol Clin 2016;34:363-74.

4 Thakkar V, Lau EMT. Connective tissue disease-related pulmonary arterial hypertension. Best Pract Res Clin Rheumatol 2016;30:22-38.

5 Chung L, Liu J, Parsons L, et al. Characterization of connective tissue diseaseassociated pulmonary arterial hypertension from reveal: identifying systemic sclerosis as a unique phenotype. Chest 2010;138:1383-94.

6 Coghlan JG, Denton CP, Grünig E, et al. Evidence-Based detection of pulmonary arterial hypertension in systemic sclerosis: the detect study. Ann Rheum Dis 2014:73:1340-9

7 Hachulla E, Carpentier P, Gressin V, et al. Risk factors for death and the 3-year survival of patients with systemic sclerosis: the French ItinérAIR-Sclérodermie study. Rheumatology 2009;48:304-8

8 Hassoun PM, Zamanian RT, Damico R, et al. Ambrisentan and tadalafil up-front combination therapy in Scleroderma-associated pulmonary arterial hypertension. Am J Respir Crit Care Med 2015;192:1102-10.

9 Galiè N, Channick RN, Frantz RP, et al. Risk stratification and medical therapy of pulmonary arterial hypertension. Eur Respir J 2019;53:1801889.

10 Galiè N, Humbert M, Vachiery J-L, et al. 2015 ESC/ERS Guidelines for the diagnosis and treatment of pulmonary hypertension. Eur Respir J 2015;46:903-75. 
11 Weatherald J, Boucly A, Launay D, et al. Haemodynamics and serial risk assessment in systemic sclerosis associated pulmonary arterial hypertension. Eur Respir J 2018;52:1800678.

12 Galiè N, Barberà JA, Frost AE, et al. Initial use of ambrisentan plus tadalafil in pulmonary arterial hypertension. N Engl J Med 2015;373:834-44.

13 Coghlan JG, Galiè N, Barberà JA, et al. Initial combination therapy with ambrisentan and tadalafil in connective tissue disease-associated pulmonary arterial hypertension (CTD-PAH): subgroup analysis from the ambition trial. Ann Rheum Dis 2017;76:1219-27

14 Hoeper MM, McLaughlin VV, Barberá JA, et al. Initial combination therapy with ambrisentan and tadalafil and mortality in patients with pulmonary arterial hypertension: a secondary analysis of the results from the randomised, controlled ambition study. Lancet Respir Med 2016;4:894-901.

15 Mathai SC, Suber T, Khair RM, et al. Health-Related quality of life and survival in pulmonary arterial hypertension. Ann Am Thorac Soc 2016;13:31-9.

16 Fisher MR, Mathai SC, Champion HC, et al. Clinical differences between idiopathic and scleroderma-related pulmonary hypertension. Arthritis Rheum 2006;54:3043-50.

17 Shapiro S, Traiger GL, Turner M, et al. Sex differences in the diagnosis, treatment, and outcome of patients with pulmonary arterial hypertension enrolled in the registry to evaluate early and long-term pulmonary arterial hypertension disease management. Chest 2012;141:363-73.

18 Weatherald J, Boucly A, Chemla D, et al. Prognostic value of follow-up hemodynamic variables after initial management in pulmonary arterial hypertension. Circulation 2018:137:693-704

19 Humbert M, Farber HW, Ghofrani H-A, et al. Risk assessment in pulmonary arterial hypertension and chronic thromboembolic pulmonary hypertension. Eur Respir J 2019;53:1802004

20 Kylhammar D, Kjellström B, Hjalmarsson C, et al. A comprehensive risk stratification at early follow-up determines prognosis in pulmonary arterial hypertension. Eur Heart $J$ 2018;39:4175-81.
21 Vachiéry J-L, Tedford RJ, Rosenkranz S, et al. Pulmonary hypertension due to left heart disease. Eur Respir J 2019;53:1801897.

22 Shirai Y, Kuwana M. Complex pathophysiology of pulmonary hypertension associated with systemic sclerosis: potential unfavorable effects of vasodilators. J Scleroderma Relat Disord 2017;2:92-9

23 Boucly A, Weatherald J, Savale L, et al. Risk assessment, prognosis and guideline implementation in pulmonary arterial hypertension. Eur Respir J 2017;50:1700889.

24 Hoeper MM, Kramer T, Pan Z, et al. Mortality in pulmonary arterial hypertension: prediction by the 2015 European pulmonary hypertension guidelines risk stratification model. Eur Respir J 2017:50:1700740.

25 Frost AE, Hoeper MM, Barberá JA, et al. Risk-Stratified outcomes with initial combination therapy in pulmonary arterial hypertension: application of the reveal risk score. J Heart Lung Transplant 2018;37:1410-7.

26 Galiè N, McLaughlin VV, Rubin LJ, et al. An overview of the 6th world Symposium on pulmonary hypertension. Eur Respir J 2019:53:1802148.

27 Cogswell R, Pritzker M, De Marco T. Performance of the reveal pulmonary arterial hypertension prediction model using non-invasive and routinely measured parameters J Heart Lung Transplant 2014;33:382-7.

28 Ghofrani H-A, Galiè N, Grimminger $\mathrm{F}$, et al. Riociguat for the treatment of pulmonary arterial hypertension. N Engl J Med 2013;369:330-40.

29 Ghofrani H-A, D'Armini AM, Grimminger F, et al. Riociguat for the treatment of chronic thromboembolic pulmonary hypertension. N Engl J Med 2013;369:319-29.

30 Rubin LJ, Galiè N, Grimminger F, et al. Riociguat for the treatment of pulmonary arterial hypertension: a long-term extension study (PATENT-2). Eur Respir $J$ 2015;45:1303-13.

31 Simonneau G, D'Armini AM, Ghofrani H-A, et al. Riociguat for the treatment of chronic thromboembolic pulmonary hypertension: a long-term extension study (CHEST-2). Eur Respir J 2015;45:1293-302.

32 Burks M, Stickel S, Galiè N. Pulmonary arterial hypertension: combination therapy in practice. Am J Cardiovasc Drugs 2018;18:249-57. 


\section{Correction: Initial combination therapy of ambrisentan and} tadalafil in connective tissue disease-associated pulmonary arterial hypertension (CTD-PAH) in the modified intention-totreat population of the AMBITION study: post hoc analysis

Kuwana M, Blair C, Takahashi T, et al. Initial combination therapy of ambrisentan and tadalafil in connective tissue disease-associated pulmonary arterial hypertension (CTD-PAH) in the modified intention-to-treat population of the AMBITION study: post hoc analysis. Ann of Rheum Dis 2020;79:626-34.

In table 1 the pvr unit should be dyne.s/cm5 and the number of FC III in SSc/Mono should be $39(70)$ not $36(77)$

\section{() \\ OPEN ACCESS}

Open access This is an open access article distributed in accordance with the Creative Commons Attribution Non Commercial (CC BY-NC 4.0) license, which permits others to distribute, remix, adapt, build upon this work noncommercially, and license their derivative works on different terms, provided the original work is properly cited, appropriate credit is given, any changes made indicated, and the use is non-commercial. See: http://creativecommons.org/licenses/by-nc/ 4.0\%.

(C) Author(s) (or their employer(s)) 2020. Re-use permitted under CC BY-NC. No commercial re-use. See rights and permissions. Published by BMJ.

Ann Rheum Dis 2020;79:e118. doi:10.1136/annrheumdis-2019-216274corr1

D) Check for updates 